\title{
Enhanced susceptibility of Cpt1c knockout mice to glucose intolerance induced by a high-fat diet involves elevated hepatic gluconeogenesis and decreased skeletal muscle glucose uptake
}

\author{
X. F. Gao $\cdot$ W. Chen $\cdot$ X. P. Kong • A. M. Xu • \\ Z. G. Wang $\cdot$ G. Sweeney $\cdot$ D. Wu
}

Received: 26 October 2008 / Accepted: 12 January 2009 / Published online: 18 February 2009

(C) Springer-Verlag 2009

\begin{abstract}
Aims/hypothesis Carnitine palmitoyltransferase-1 (CPT1)c is a novel isoform in the CPT1 family and is found specifically in the brain. Cptlc knockout (KO) mice are more susceptible to high-fat diet (HFD)-induced obesity. However, the underlying mechanism of this phenotype and the question of whether CPT1c is involved in the pathogenesis of diet-induced insulin resistance are unclear.

Methods To assess the potential role of CPT1c in the regulation of whole-body glucose homeostasis, we generated Cpt1c KO mice and challenged them with HFD or standard chow. Glucose homeostasis of each group was assessed weekly.
\end{abstract}

Electronic supplementary material The online version of this article (doi:10.1007/s00125-009-1284-0) contains supplementary material, which is available to authorized users.

X. F. Gao $\cdot$ W. Chen $\cdot$ A. M. Xu • D. Wu $(\bowtie)$

Key Laboratory of Regenerative Biology,

Guangzhou Institute of Biomedicine and Health,

Chinese Academy of Sciences,

Guangzhou, China 510663

e-mail: wu_donghai@gibh.ac.cn

X. P. Kong

458th Hospital,

Guangzhou, China

A. M. Xu

Department of Medicine, University of Hong Kong,

Hong Kong, China

Z. G. Wang

Shanghai Nan Fang Model Organism Research Center,

Shanghai, China

G. Sweeney

Department of Biology, York University,

Toronto, Canada
Results After 8 weeks of HFD feeding, Cpt1c KO mice developed a phenotype of more severe insulin resistance than that in wild-type controls. The increased susceptibility of Cpt1c KO mice to HFD-induced insulin resistance was independent of obesity. Impaired glucose tolerance in Cpt1c KO mice was attributable to elevated hepatic gluconeogenesis and decreased glucose uptake in skeletal muscle. These effects correlated with decreased hepatic and intramuscular fatty acid oxidation and expression of oxidative genes as well as with elevated triacylglycerol content in these tissues. Interestingly, Cpt1c deletion caused a specific elevation of hypothalamic CPT1a and CPT1b isoform expression and activity. We demonstrated that elevated plasma NEFA concentration is one mechanism via which this compensatory effect is induced.

Conclusions/interpretation These results further establish the role of CPT1c in controlling whole-body glucose homeostasis and in the regulation of hypothalamic Cpt1 isoform expression. We identify changes in hepatic and skeletal muscle glucose metabolism as important mechanisms determining the phenotype of Cptlc KO mice.

Keywords CPT1 activity Cpt1 expression .

Cpt1c knockout · Fatty acid oxidation - Gluconeogenesis .

Glucose intolerance - Glucose uptake · Hypothalamus .

Non-esterified fatty acid · Oxidative genes expression

$\begin{array}{ll}\text { Abbreviations } \\ \text { CNS } & \text { Central nervous system } \\ \text { CPT1 } & \text { Carnitine palmitoyltransferase-1 } \\ \text { GTT } & \text { Glucose tolerance test } \\ \text { HFD } & \text { High-fat diet } \\ \text { ITT } & \text { Insulin tolerance test } \\ \text { KO } & \text { Knockout } \\ \text { N2a } & \text { Neuroblastoma }\end{array}$




\section{RNAi RNA interference}

shRNA Short-hairpin RNA

WT Wild-type as changes in Cpt1 expression and activity in the CNS of these $\mathrm{KO}$ mice.

\section{Methods}

Targeting Cpt1c allele A mouse clone was isolated by screening a library made with genomic DNA from a $129 \mathrm{~Sv}$ mouse strain with a cDNA encoding murine Cpt1c as a probe. A $6.7 \mathrm{~kb}$ fragment of the mouse Cptlc genome containing coding exons 1 to 6 was used to construct a targeting vector and a $1.3 \mathrm{~kb}$ fragment containing the sequence of exon 3 was deleted. The targeting vector also included a positive neomycin ( $\mathrm{Neo})-P g k$ selection cassette and a negative $P g k$-diphtheria toxin $(D T)$ selection cassette. We transfected $129 \mathrm{~Sv}$ embryonic stem cells with a linearised targeting vector by electroporation. After selection, clones with a targeted allele were identified by PCR analysis. Embryonic stem cells with the targeted allele were injected into blastocysts of $\mathrm{C} 57 \mathrm{BL} / 6 \mathrm{~J}$ mice and chimeric males were mated with females of the same strain to obtain heterozygous mutant mice, which were then intercrossed to obtain homozygous and wild-type (WT) mice. The frequency of homozygous, WT and heterozygous pups in the offspring of heterozygous parents reflected a Mendelian distribution. Male mice were chosen for further analysis as detailed below. All experiments with animals were approved by the Animal Care and Use Committee of Guangzhou Institute of Biomedicine and Health, Chinese Academy of Sciences.

Standard chow and HFD feeding Male mice (7 weeks old) were housed in metabolism cages and maintained on a $12 \mathrm{~h}$ light-dark cycle at $23^{\circ} \mathrm{C}$ and fed standard chow $(15.9 \mathrm{~kJ} / \mathrm{g}$, $10 \%$ of energy as fat, $20 \%$ of energy as protein, $70 \%$ of energy as carbohydrate; GB14924; Guangdong Medical Laboratory Animal Center, Guangzhou, China) or HFD $(21.9 \mathrm{~kJ} / \mathrm{g}, 60 \%$ of energy as fat, $20 \%$ of energy as protein, $20 \%$ of energy as carbohydrate; D12492; Research Diet, New Brunswick, NJ, USA) according to the experimental design. Food intake was measured daily and body weight was measured weekly during the experimental phase in all experiments. For body temperature measurement, mice were held loosely in a small cloth bag and the core body temperature was measured using a digital thermometer with a resolution of $0.1{ }^{\circ} \mathrm{C}$ (MT-132; Mother Tool, Ueda, Japan). The thermometer probe was inserted $25 \mathrm{~mm}$ into the rectum.

Plasma constituents analyses Blood samples were taken from cut tail tips of conscious overnight fasted mice. Plasma was collected for measurement of constituents. Plasma triacylglycerol and cholesterol were measured using commercial kits (Triacylglycerol Kit and Cholesterol Kit; and oxidative gene expression in peripheral tissue, as well 
Jiancheng, Nanjing, China). Plasma leptin was measured by a kit (Mouse/Rat leptin ELISA kit; Biovendor, Modrice, the Czech Republic).

Metabolic analyses; glucose and insulin tolerance tests A bolus of glucose ( $2 \mathrm{~g} / \mathrm{kg}$ of body weight) or insulin $(0.5 \mathrm{U} / \mathrm{kg}$ of body weight) was administered by intraperitoneal injection to mice previously fasted for $6 \mathrm{~h}$ (from 09:00 to 15:00 hours). Blood glucose was monitored from the tail vein blood using a glucometer (ACCU-CHEK Advantage; Roche Diagnostics China, Shanghai, China) before or 15, 30, 60 and $120 \mathrm{~min}$ after glucose or insulin administration. Insulin level was measured by a mouse insulin ELISA kit (Mercodia, Uppsala, Sweden). The pyruvate tolerance test was performed as described [26, 27]. For plasma NEFA analysis, food was removed from each group at 08:00 hours and plasma NEFA concentrations at $0,1,2,6$ and $12 \mathrm{~h}$ after food removal were monitored from tail vein blood using a commercial kit (NEFA Kit; Jiancheng).

Metabolic analysis in tissues The method for tissue triacylglycerol extraction and determination was as described previously $[28,29]$. Glycogen analysis was performed using a commercial kit (Glycogen Kit; Jiancheng). Glucose uptake analysis of skeletal soleus muscle in vivo or in vitro was determined as described [30, 31]. Measurement of fatty acid oxidation of liver and skeletal soleus muscle in vitro was performed as described [32] with slight modification. Details on tissue analysis are given in the Electronic supplementary material (ESM).

RNA interference strategy to knock down CPT1c in neuroblastoma cells We used a vector-based RNA interference (RNAi) approach to produce intracellularly a doublestranded short hairpin RNA (shRNA) from a DNA template under the control of the U6.2 promoter. Annealed oligonucleotides were cloned into the BamHI-XhoI sites of the pRNAT-U6.2/Lenti vector (GenScript, Piscataway, NJ, USA) and were designed to target the coding sequence of Cptlc from site 21 to 50. The sequence of the oligonucleotides was: 5'-GGATCCCGTCGGAACTCAGTGAAGAC A GCA A GC T C G AT T GATAT C C G T C GA G C T T GCTGTCTTCACTGAGTTCCGATTTTTTCCAACTC GAG - $3^{\prime}$. Stable cells with Cpt1c shRNA expression were used for further analysis (for details see ESM). Palmitate (Sigma, St Louis, MO, USA) was dissolved in 95\% ethanol (vol./vol.) at $60^{\circ} \mathrm{C}$ and then mixed with pre-warmed BSA (molar ratio: $4: 1$ ) to yield a stock concentration of $10 \mathrm{mmol} / \mathrm{l}$. Palmitate was added to the medium and cells were collected for analysis $10 \mathrm{~h}$ later.

Measurement of CPT1 activity Mitochondria of muscle, liver, hypothalamus and neuroblastoma (N2a) cells were prepared as described previously [16, 20, 33], protein concentrations determined using the Bradford assay and CPT1 activity assayed as described [34, 35] with slight modification. The details are given in the ESM.

Gene expression analysis Total RNA was isolated from cells or tissues of mice fasted for $6 \mathrm{~h}$ using Trizol reagent (Invitrogen China, Guangzhou, China) and isolated total RNA was reverse-transcribed with Superscript III reverse transcriptase (Invitrogen). For real-time PCR analysis, cDNA samples were used in quantitative PCR reaction in the presence of fluorescent dye Cybergreen (Bio-Rad, Benicia, CA, USA). The following PCR conditions were applied: $5 \mathrm{~min}, 95^{\circ} \mathrm{C} ; 40 \times\left(10 \mathrm{~s}, 95^{\circ} \mathrm{C} ; 20 \mathrm{~s}, 60^{\circ} \mathrm{C} ; 1 \mathrm{~s}, 70^{\circ}\right.$ C). After each elongation step, the reaction was quantified in a reading step and the product quality tested by melting curve analysis. Relative abundance of mRNA was calculated after normalisation to Gapdh mRNA. Sequences for the primers used in this study are shown in ESM Table 1.

Western blot analysis CPT1c immunoblotting in tissue or cells was done using polyclonal CPT1c antibody, which was produced in rabbits against the peptide CKTVDP NTPTSSTNL coupled with keyhole limpet haemocyanin. The antibody was tested against epitope-tagged mouse CPT1c and mouse CPT1a constructs and shown to be specific to CPT1c.

Statistical analysis Data are expressed as mean \pm SEM. All comparisons were analysed by unpaired, two-tailed Student's $t$ test. Pearson correlation coefficient was used to establish the association between plasma NEFA concentration and both increased CPT1 activity and Cpt 1 mRNA fold change in hypothalamus. A $p$ value of $p<0.05$ was considered significant.

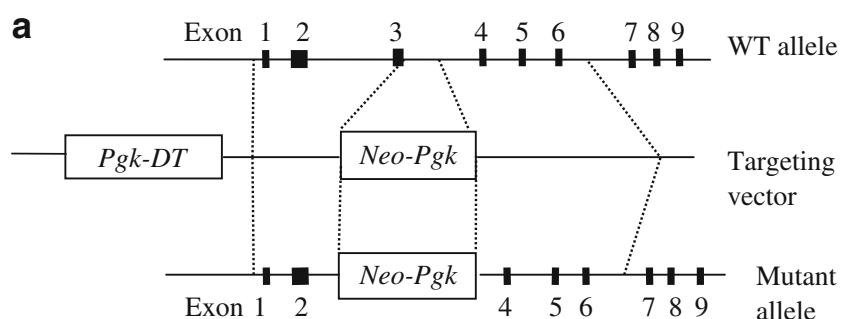

b

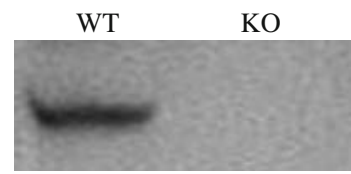

Fig. 1 Targeted disruption of mouse Cpt1c gene. a Genomic locus, targeting vector and predicted targeting locus. b The CPT1c protein in brain of WT and KO mice was analysed by western blot. DT, diphtheria toxin 


\section{Results}

Generation of Cptlc KO mice To study the physiological role of CPT1c in vivo, we produced a disruption of Cpt1c using a targeting vector designed to replace exon 3 with Neo-Pgk gene cassette (Fig. 1a). The genotype of these offspring was identified by tail genome PCR (ESM Fig. 1). Western blot analysis in whole brain showed that the Cpt1c KO mice were completely deficient in CPT1c protein (Fig. 1b). These mice were then used for metabolic analyses. Wolfgang et al. also recently generated Cptlc KO mice using a different genetic strategy [20]. Importantly, our Cptlc KO mice display a similar basic phenotype to that of previously described mice, thus confirming the role of $\mathrm{CPT} 1 \mathrm{c}$ in regulating peripheral glucose homeostasis. In this study we performed a detailed mechanistic analysis of this phenomenon. Some important differences between our approach and that of Wolfgang et al. are outlined in Table 1.

Cptlc KO mice are more susceptible to HFD-induced insulin resistance To determine whether CPT1c was involved in the onset of diet-induced insulin resistance, we fed the Cptlc KO and WT mice standard chow or HFD and assessed glucose homeostasis in each group weekly. After 8 weeks of feeding, the body fat mass of HFD Cptlc KO and WT mice was markedly increased compared with standard chow controls, while there was no significant difference between Cptlc KO and WT mice in HFD and standard chow groups (ESM Table 2). No differences in fasting glucose and insulin levels were observed between the two types of mice under standard chow condition (ESM Fig. 2a, b). However, Cpt1c KO mice showed significantly higher fasting glucose and insulin levels than WT mice on HFD (ESM Fig. 2a, b). To further assess insulin sensitivity of each group, we performed a glucose tolerance test (GTT) and insulin tolerance test (ITT). As illustrated in Fig. 2a, b, HFD significantly decreased the insulin sensitivity of each

Table 1 Differences in experimental strategy and generation of Cpt1c $\mathrm{KO}$ mouse model for this study compared with a previous analysis [20]

\begin{tabular}{lll}
\hline Items & Our model & Previous model \\
\hline Genetic background (\%) & 129Sv (50)/ & C57BL/6J \\
& C57BL/6J (50) & $\begin{array}{c}(100) \\
\text { Exon 3 }\end{array}$ \\
KO strategy & 60 & 45 \\
HFD used (\% energy from fat) & 60 \\
Age (weeks) at exposure to HFD $\approx 7$ & $\approx 15$ \\
Pathogenic timing (weeks after & 8 & 17 \\
HFD feeding) & No & Yes \\
Obesity state vs WT &
\end{tabular}

mouse type. Specifically, compared with WT HFD mice, Cpt1c KO mice on HFD developed more severe insulin resistance. The AUC of GTTs and ITTs further supported the significant difference between $\mathrm{KO}$ and WT mice on HFD (Fig. 2c). We also showed that the glucose intolerance of WT mice after 24 weeks of HFD feeding was worsened
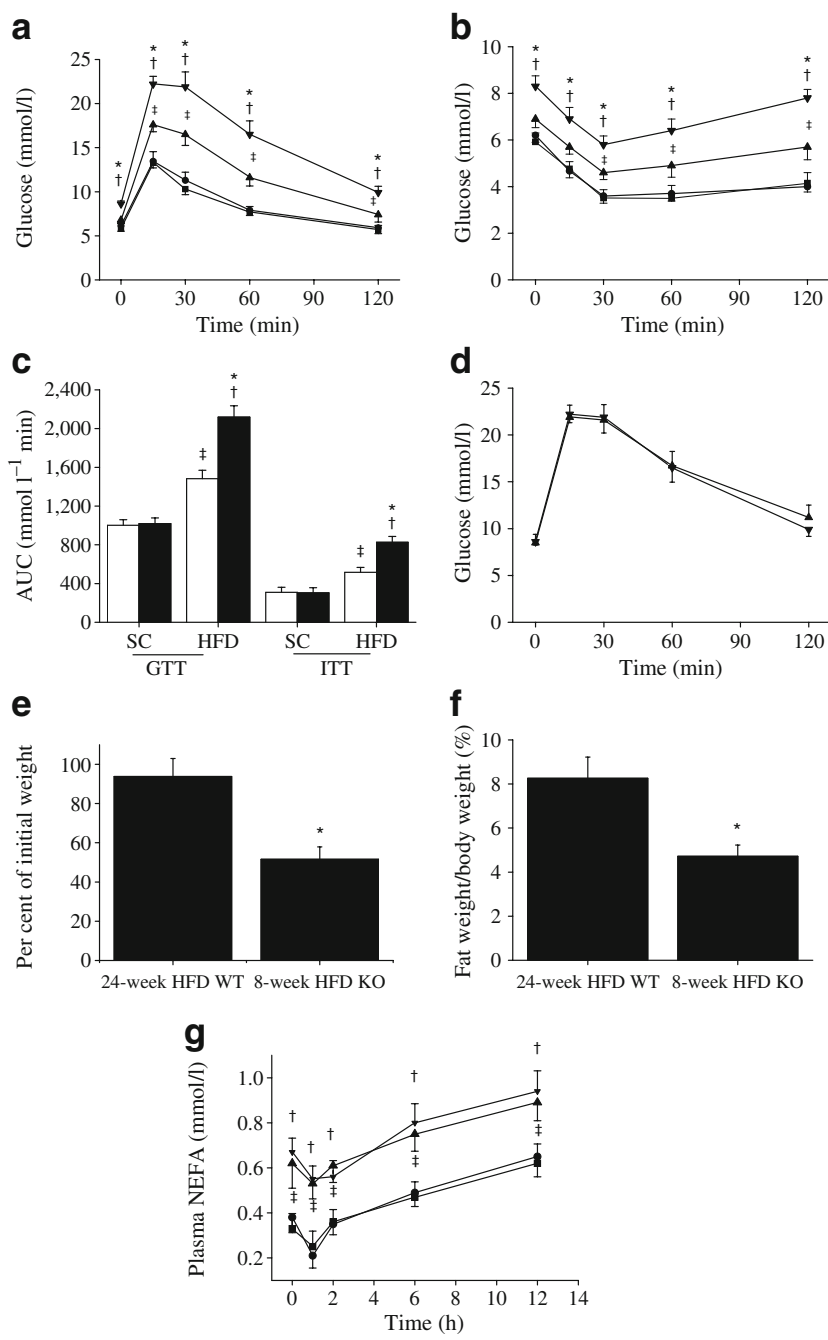

Fig. 2 In vivo glucose homeostasis and insulin sensitivity of each mouse group. Glucose homeostasis in each group after 8 week on HFD or standard chow (SC) was determined by GTT (a) and ITT (b) $(n=12)$. Squares, SC WT; circles, SC KO; upright triangles, HFD WT; inverted triangles, HFD KO. The AUC of GTT and ITT was also calculated (c). White bars, WT; black bars, KO. Comparison of GTT (upright triangles, 24 week HFD WT; inverted triangles, 8 week HFD KO) (d), bodyweight gain (e) and fat (epididymal and inguinal adipose tissue) as percentage of body weight (f) between Cpt1c KO mice on 8 weeks of HFD and WT mice on 24 weeks of HFD were analysed $(n=9-12)$. g Plasma NEFA concentrations of mice on 8-week HFD and standard chow were measured over a period of $12 \mathrm{~h}$ following food removal $(n=$ $7-9)$, key as above (a, b). Values are means \pm SEM. ${ }^{*} p<0.05$ compared with WT mice on HFD; ${ }^{\dagger} p<0.05$ compared with KO mice on standard chow; ${ }^{*} p<0.05$ compared with WT mice on standard chow 
to the same extent as that of KO mice after 8 weeks of HFD feeding, despite a significant increase in body weight gain and body fat (Fig. 2d-f). Notably, in both types of mice on HFD circulating NEFA increased significantly (Fig. 2g).

Cpt1c KO mice on HFD exhibited increased gluconeogenesis To determine gluconeogenesis in vivo, we measured the blood glucose levels of mice following intraperitoneal injection of pyruvate, an important substrate of gluconeogenesis. The results showed that HFD feeding enhanced the rate of pyruvate conversion into glucose and that $C p t 1 c \mathrm{KO}$ mice on HFD exhibited a higher converting rate than WT mice under the same feeding regimen (Fig. 3a). mRNA levels of hepatic glucoregulatory genes G6pc and Pckl were significantly increased in WT mice on HFD and to an even greater degree in Cpt1c KO mice (Fig. 3b). Liver glycogen content of these two types of mice on HFD was a

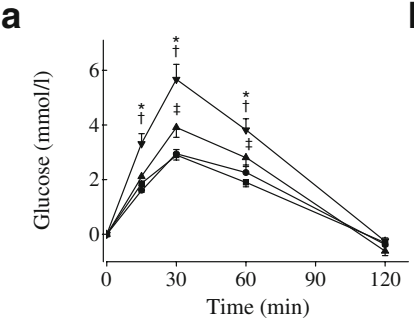

d

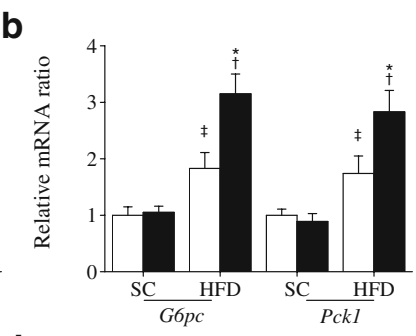

d

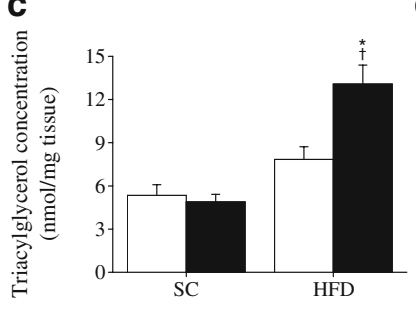

e

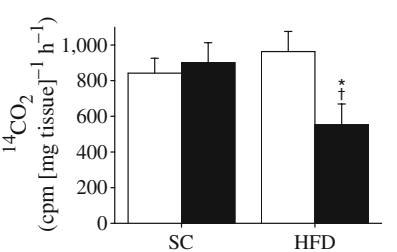

f

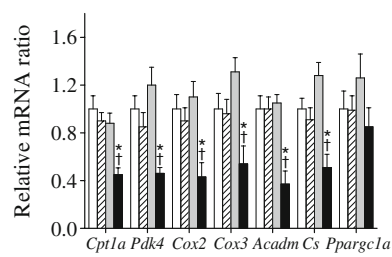

Fig. 3 Increased gluconeogenesis of Cptlc KO mice on HFD. a The in vivo gluconeogenesis of each group after 8 weeks of HFD feeding was examined by intraperitoneal injection of pyruvate and the increase in plasma glucose concentration from baseline (set to 0$)(n=12)$. Squares, SC WT; circles, SC KO; upright triangles, HFD WT; inverted triangles, HFD KO. b Real-time PCR analysis of two glucoregulatory enzymes, including $P c k 1$ and $G 6 p c$ mRNA expression, in liver isolated from each mouse type fasted for $6 \mathrm{~h}$. c Triacylglycerol content, CPT1 activity (d) and the fatty acid oxidation (e) in liver $(n=6)$. White bars, WT; black bars, KO. f Expression of some oxidative genes, analysed by real-time PCR $(n=6)$. White bars, standard chow WT; hatched bars, standard chow KO; grey bars, HFD WT; black bars, HFD KO. Values are means \pm SEM. ${ }^{*} p<0.05$ compared with WT mice on HFD $;{ }^{\dagger} p<0.05$ compared with KO mice on SC; ${ }^{\dagger} p<0.05$ compared with WT mice on SC similar (ESM Fig. 2c), suggesting that glycogenolysis is not an important factor contributing to the increased endogenous glucose levels of Cpt1c KO mice on HFD. However, liver triacylglycerol content was increased in $\mathrm{KO}$ mice fed HFD (Fig. 3c), which was associated with significant decreased total CPT1 activity (Fig. 3d) and fatty acid oxidation (Fig. 3e). Consistent with this, the expression of genes promoting fatty acid oxidation and energy metabolism, including Cpt1a, Acadm, Pdk4, Cox2, Cox3 and Cs, was markedly decreased in Cpt1c KO mice on HFD compared with WT mice (Fig. 3f).

Cpt1c KO mice on HFD showed decreased glucose uptake in skeletal muscle To assess whether alterations in peripheral glucose uptake are also responsible for the impaired glucose tolerance in Cpt1c KO mice on HFD, we analysed glucose uptake in vivo. After 8 weeks of HFD feeding, glucose uptake in skeletal muscle was considerably decreased in Cpt1c KO mice on HFD compared with WT controls (Fig. 4a). Glucose uptake in white adipose and brown adipose tissue of Cpt1c KO and WT mice was significantly decreased compared with standard chow controls, while there was no significant difference between $C p t 1 c$ KO HFD mice and WT controls (data not shown). The isolated skeletal muscle was used to further analyse the glucose uptake defect in muscle of Cpt1c KO mice on HFD. The results showed that under insulin stimulation, the glucose uptake capacity was significantly decreased in isolated muscle of Cpt1c KO mice on HFD compared with that of WT mice, while there was no significant difference in non-insulin-stimulated glucose uptake among each of these groups (Fig. 4b). To eliminate the effect of glucose 6-phosphatase on the accumulation of 2-deoxy $\left[{ }^{3} \mathrm{H}\right]$ glucose6-phosphate, we examined the G6pc mRNA level in skeletal muscle of each group of mice. In contrast to liver, the mRNA levels of G6pc in skeletal muscle of Cpt1c KO HFD mice and WT controls were similar (ESM Fig. 3a). A significantly reduced glycogen content and elevated triacylglycerol content were observed in skeletal muscle of $C p t 1 c$ KO mice on HFD (Fig. 4c, d), with no significant difference detected between Cpt1c KO and WT mice on standard chow. Also, these alterations were correlated with decreases in CPT1 activity and fatty acid oxidation in muscle (Fig. 4e, f). These results are consistent with the downregulation of oxidative genes expression, including Cpt1b, Acadm, Pdk4, Cox2, Cox3, Cs and Ppargcla, seen in Cpt1c KO mice on HFD compared with the WT mice (Fig. 4g).

Hypothalamic CPT1 activity was increased in Cpt1c KO mice on HFD All three members of the CPT1 family are expressed in the hypothalamus. Therefore, we sought to examine whether Cpt1c deletion alters Cpt1a or Cpt1b expression and activity in the hypothalamus. We found a 
a

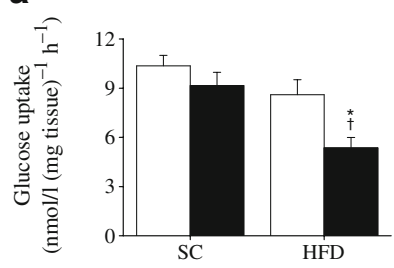

c

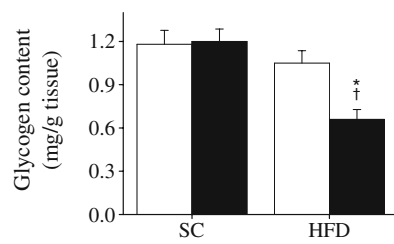

e

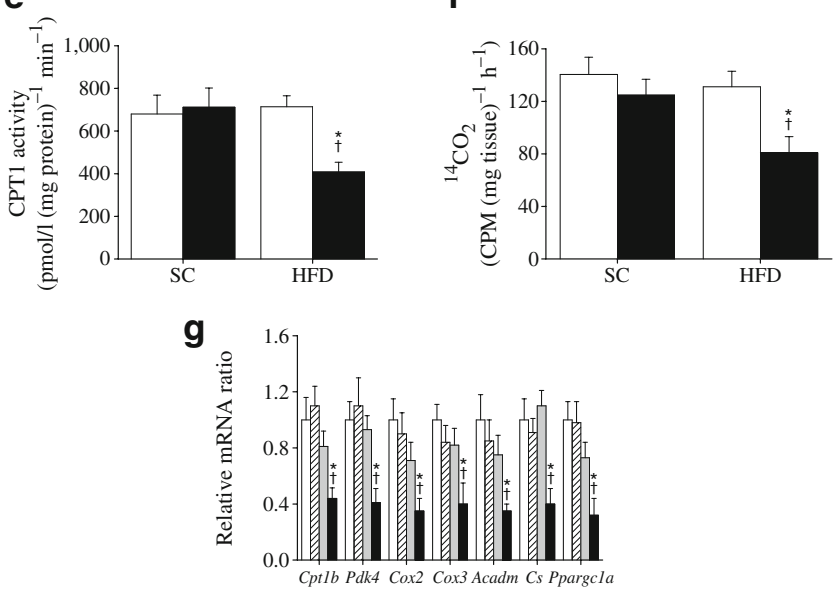

Fig. 4 Attenuated skeletal muscle glucose uptake of Cpt1c KO mice on HFD. a Glucose uptake in vivo and (b) in vitro in skeletal soleus muscle of mice at 8 weeks feeding and fasted for $6 \mathrm{~h}$ was determined and normalised to tissue weight. c Glycogen, (d) triacylglycerol content, (e) CPT1 activity and (f) fatty acid oxidation in soleus muscle were determined. White bars, WT; black bars, KO. g Expression of oxidative genes was analysed by real-time PCR. White bars, standard chow WT; hatched bars, standard chow KO; grey bars, HFD WT; black bars, HFD KO. Values are means \pm SEM for five to six mice per group, ${ }^{*} p<0.05$ compared with WT mice on HFD; ${ }^{\dagger} p<0.05$ compared with KO mice on standard chow; ${ }^{\ddagger} p<0.05$ compared with WT mice on standard chow

substantial increase in the hypothalamic mRNA levels of Cptla and Cpt1b in Cpt1c KO mice on HFD compared with those in WT mice; there was no difference between these two types of mice on standard chow (Fig. 5a). Cpt1c expression in the hypothalamus of WT mice was not changed by 8 weeks of HFD feeding (ESM Fig. 3b, c). We did not observe any marked difference of Cpt1a and Cpt1b transcript levels in other CPT1c-rich regions between both Cpt1c KO and WT mice on HFD or those on standard chow (data not shown). Consistent with this, a considerably increased hypothalamic CPT1 activity in Cpt1c KO mice on HFD was observed compared with WT mice (Fig. 5b). CPT1 activity in hippocampus and cortex of a

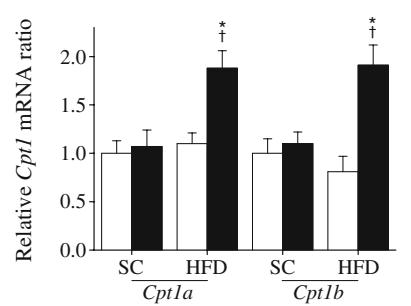

b

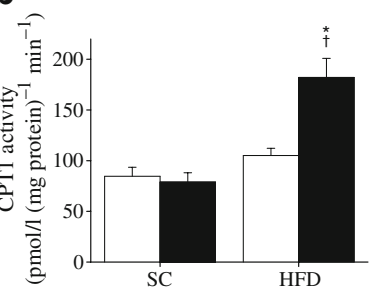

C

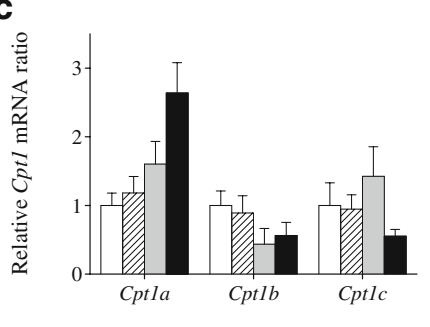

d

e

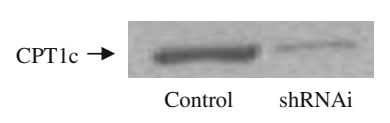

f
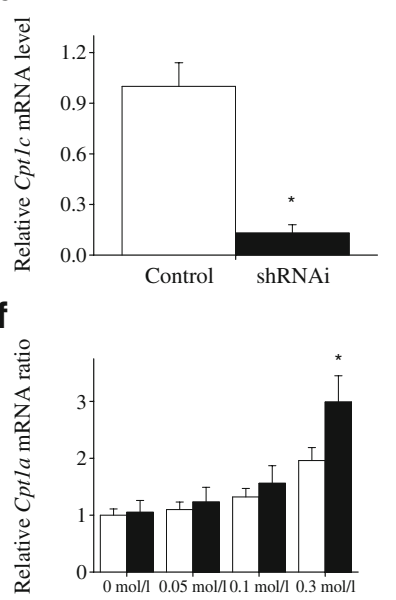

g

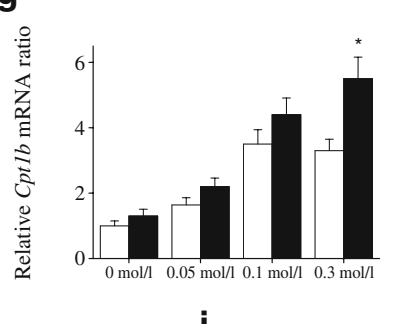

h

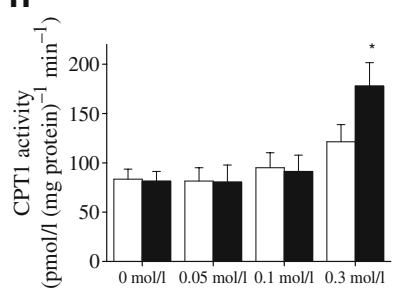

i

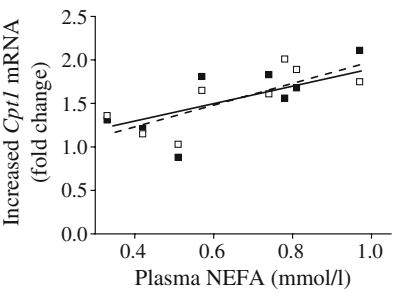

Cpt1c KO and WT mice on HFD or standard chow was similar (data not shown).

NEFA induced Cpt1a and Cpt1b expression in Cpt1c knockdown neuroblastoma To further investigate the underlying mechanism of the hypothalamus-specific compensatory effect, we selected the N2a cell as a tool. Real-time PCR analyses showed that all the Cpt1 members were expressed in N2a cells and that the Cpt1 expression profile of N2a cells was very similar to that of hypothalamus (Fig. 5c), indicating that N2a cell is a suitable in vitro model to examine the effect of Cpt1c deficiency on Cpt1a and Cpt $1 b$ expression in hypothalamus. To this end, we 
Fig. 5 Increased $C p t 1$ expression in hypothalamus of $C p t 1 c$ KO mice on HFD. Total RNA isolated from hypothalamus of mice on HFD for 8 weeks was subjected to real-time PCR analysis with primers for Cpt1a and Cpt1b (a). b The total CPT1 activity of hypothalamic mitochondrial protein was determined. White bars, WT; black bars, $\mathrm{KO}$; SC, standard chow. Values are means \pm SEM for six mice per group. ${ }^{*} p<0.05$ compared with WT mice on HFD; ${ }^{\dagger} p<0.05$ compared with KO mice on standard chow. c The Cptl expression profile analysis of N2a (white bars), hypothalamus (hatched bars), hippocampus (grey bars) and cortex (black bars) was performed $(n=4)$. d The effect of shRNAi knockdown (black bars; white bars, control) on Cpt1c expression was analysed by real-time PCR and (e) western blot. f The Cptla and Cpt1b (g) mRNA expression in shRNAi knockdown (black bars) and control cells (white bars) treated with different concentrations $(\mathrm{mmol} / \mathrm{l})$ of palmitate were analysed by realtime PCR and (h) the corresponding CPT1 activity was determined. Values are means \pm SEM for three determinations. ${ }^{*} p<0.05$ compared with the shRNAi control. i Correlation between plasma NEFA concentration and the increased Cptl mRNA fold change in hypothalamus of $C p t 1 c \mathrm{KO}$ mice compared with WT mice. Black symbols, Cpt1a, $n=8, r=0.74, p<0.05$; white symbols, $C p t 1 b, n=8$, $r=0.71, p<0.05$

reduced Cpt1c expression in N2a cell by shRNAi knockdown (Fig. 5d, e). We postulated that the increased plasma NEFA concentrations of the HFD feeding group could induce Cpt1 expression in hypothalamus of Cpt1c-deficient mice. No significant difference between Cptla and Cpt1b was observed in Cptlc shRNAi cells and control treatment when these cells were maintained in palmitate-free or lower palmitate medium (Fig. 5f, g). However, when the palmitate concentration in medium was increased to $0.3 \mathrm{mmol} / \mathrm{l}$, Cptla and Cpt1b expression in Cptlc shRNAi cells was increased significantly compared with control (Fig. 5f, g), while palmitate treatment did not change Cptlc expression significantly in control cells (ESM Fig. 3d, e). Analysis of corresponding total CPT1 activity under these conditions confirmed the ability of palmitate to significantly induce CPT1 activity at $0.3 \mathrm{mmol} / 1$ in cells lacking Cpt1c (Fig. $5 \mathrm{~h})$. This point is reinforced by the clear positive correlation between circulating NEFA and hypothalamic Cpt1 expression (Fig. 5i) and activity (ESM Fig. 4).

\section{Discussion}

Previous studies have established that lipid metabolism in the CNS is an important mechanism regulating maintenance of systemic glucose homeostasis. CPT1, the rate-limiting enzyme in fatty acid oxidation, along with its allosteric inhibitor malonyl-CoA and its substrate long-chain fatty acid-CoA, constitutes an intricate sensing feedback loop, which can regulate metabolic processes in response to the changing nutrient status of the body [3, 15-17, 32]. However, it has been demonstrated that lipid metabolism in CNS and in peripheral tissues has opposing physiolog- ical effects despite sharing similar biochemical/molecular mechanisms [16, 17, 36-38]. Specifically, inhibition of CPT1 in the hypothalamus suppresses glucose production in liver [15, 16], while inhibition of liver CPT1 with etomoxir induces liver insulin resistance [37]. The central administration of $\mathrm{C} 75$, a potent inhibitor of fatty acid synthase, increases malonyl-CoA concentration in the hypothalamus, while activating fatty acid oxidation in skeletal muscle [32, 36].

CPT1c is a novel brain-specific CPT1 isoform. Previous reports have shown that $\mathrm{CPT} 1 \mathrm{c}$ had no enzyme activity in yeast or HEK293T cells with palmitoyl-CoA or other acylCoA molecules as substrate, and that CPT1c is localised in mitochondria [18, 20, 25]. However, a recent study demonstrated CPT1c localisation in the endoplasmic reticulum of the neuron and also that CPT1c has palmitoylcarnitine-specific transferase activity, albeit much lower than other CPT1 isoforms [21]. Studies by Wolfgang et al. [20], and now ourselves using independently generated Cpt1c KO mice, have demonstrated that these mice display higher susceptibility to HFD-induced obesity and insulin resistance. Our current study was designed to gain insight into the mechanisms via which Cptlc deficiency causes greater susceptibility to HFD-induced insulin resistance and is clearly distinct yet complementary to the previous study of Cpt1c deletion as outlined in Table 1. Although the genetic background of mice in our study is different from that of the previous study [20], a similar susceptibility of Cpt1c KO mice to HFD-induced obesity was also observed in our study (ESM Fig. 5a-c), indicating that genetic background has no major effect on the physiological role of Cpt1c. In our study, after 8 weeks of feeding, the $C p t 1 c \mathrm{KO}$ mice on HFD exhibited an apparently more severe insulin resistance than WT control mice, even before body fat mass or plasma NEFA diverged significantly. The glucose intolerance of HFD Cptlc KO mice at 8 weeks of HFD feeding was similar to that of WT mice at 24 weeks of HFD feeding, despite a significant decrease in bodyweight gain and body fat. These data imply that $C p t 1 c \mathrm{KO}$ mice were more susceptible than WT controls to impaired insulin resistance and that this was independent of obesity. Additionally, our results indicate that the impaired glucose tolerance in Cptlc KO mice on HFD could be, at least in part, explained by increased hepatic gluconeogenesis and decreased muscle glucose uptake. The elevated gluconeogenesis was illustrated by the enhanced converting rate of pyruvate to glucose and by elevated expression of $G 6 p c$ and Pck1. The decreased glucose uptake in skeletal muscle also showed the contribution of skeletal muscle to the phenotype of $C p t 1 c \mathrm{KO}$ mice on HFD. In this regard, the decreased glycogen content in skeletal muscle of Cptlc $\mathrm{KO}$ mice on HFD is likely to be due to decreased insulin-stimulated glycogen synthase activity and glycogen synthesis [39, 40]. 
In this study, we also report that Cpt1c KO mice fed on HFD exhibited elevated levels of the other two functional CPT1 isoforms, CPT1a and CPT1b, specifically in hypothalamus. This was not observed in Cpt1c KO mice on standard chow or in WT mice on HFD and standard chow. The increased plasma NEFA concentration of the HFD feeding group was identified as the possible causative factor of the hypothalamic Cpt1 compensatory effect. Hypothalamus is the most important brain region for sensing circulating nutritional NEFA levels and in turn modulates global glucose homeostasis $[16,41]$; in fact, in our current study the same compensatory effects were not found in other brain regions. Moreover, various brain regions exhibit different lipid metabolism profiles under same treatment conditions $[15,16]$. As a critical regulatory factor of lipid metabolism, the Cpt1 expression profile is quite different in various brain regions and these differences have been shown to be correlated with different metabolic responses to circulating NEFA even between two brain regions that are very close together $[15,16]$. Hence, we selected the N2a cell, which exhibits similar Cpt1 expression profile to hypothalamus, to investigate the underlying mechanism of this hypothalamic compensatory effect. Indeed, our demonstration that NEFA can induce Cpt 1 expression in this cell line and the correlation of circulating NEFA with hypothalamic Cpt1 expression and activity confirm this potential mechanism.

We also detected decreased fatty oxidation and oxidative genes expression in liver and skeletal muscle and so it appears that the opposing responses of oxidative enzymatic activity in central and peripheral systems ultimately contribute to impaired glucose tolerance [17, 37, 42]. This could be the primary defect, from which the metabolic disturbance of Cpt1c KO mice originates. Therefore, the compensatory effect of functional Cptl in hypothalamus and the adverse effect of decreased peripheral energy metabolism in $C p t 1 c \mathrm{KO}$ mice provide a possible, initial mechanistic explanation of the phenotype of Cpt1c KO mice on HFD while confirming an important function of this brain-specific CPT1 isoform in whole-body energy regulation. Although the CPT1 activity of CPT1c is very low, the capacity of CPT1c to bind malonyl-CoA is similar to that of CPT1a [20]. Thus, we hypothesise that hypothalamic CPT1c binds malonyl-CoA and thus, through this binding, regulates the availability of intracellular malonylCoA. Deficiency of CPT1c makes more malonyl-CoA available, especially under HFD conditions; as a result, the subsequently decreased CPT1 activity indicates that fatty acid oxidation was blunted, with substrate accumulation possibly inducing functional CPT1 production in hypothalamus via a compensatory feedback mechanism. Clearly, additional rigorous experimentation is needed to validate this hypothesis. Similarly, further dedicated studies will be necessary to define whether the peripheral metabolic changes are the results of CPT1c deficiency or the secondary consequence of increased functional $\mathrm{Cpt} 1$ expression in CNS.

In summary, we have shown that under HFD feeding conditions, Cpt1c KO mice were more susceptible to HFDinduced insulin resistance. This manifested as defects in gluconeogenesis and muscle glucose uptake and could be partially attributed to defective energy metabolism in these tissues. Consistent with the peripheral hyperglycaemia in these mice, we further observed a compensatory elevation of two other CPT1 isoforms in the hypothalamus of Cpt1c KO mice on HFD and identified increased plasma NEFA concentration as the possible causative event for this compensation. These results indicate a possible function of CPT1c in regulating energy homeostasis during times of energy surplus.

Acknowledgements This work was supported in part by funds from: the National Science Foundation of China (30670457, 90813033 and 30811120429), Guangzhou Administration of Science and Technology (2007Z2-E4021 and 2005Z3-C7181), Guangzhou Economic and Technological Development District matching funds (2007Ss-P059) and the National 973 Programme of China (2007CB914301, 2006CB910202, 2006CB503908 and 2004CB720102). G. Sweeney thanks the Canadian Institutes of Health Research for research support.

Duality of interest statement The authors declare that there is no duality of interest associated with this manuscript.

\section{References}

1. Flegal KM, Carroll MD, Ogden CL, Johnson CL (2002) Prevalence and trends in obesity among US adults, 1999-2000. JAMA 288:1723-1727

2. Finer N (2003) Obesity. Clin Med (London) 3:23-27

3. Wolfgang MJ, Lane MD (2006) The role of hypothalamic malonyl-CoA in energy homeostasis. J Biol Chem 281:3726537269

4. Shimokawa T, Kumar MV, Lane MD (2002) Effect of a fatty acid synthase inhibitor on food intake and expression of hypothalamic neuropeptides. Proc Natl Acad Sci U S A 99:66-71

5. Pocai A, Lam TK, Gutierrez-Juarez R et al (2005) Hypothalamic K(ATP) channels control hepatic glucose production. Nature 434:1026-1031

6. Plum L, Belgardt BF, Bruning JC (2006) Central insulin action in energy and glucose homeostasis. J Clin Invest 116:1761-1766

7. Minokoshi Y, Alquier T, Furukawa N et al (2004) AMP-kinase regulates food intake by responding to hormonal and nutrient signals in the hypothalamus. Nature 428:569-574

8. Lam TK, Pocai A, Gutierrez-Juarez R et al (2005) Hypothalamic sensing of circulating fatty acids is required for glucose homeostasis. Nat Med 11:320-327

9. Thupari JN, Landree LE, Ronnett GV, Kuhajda FP (2002) C75 increases peripheral energy utilization and fatty acid oxidation in diet-induced obesity. Proc Natl Acad Sci U S A 99:9498-9502

10. Loftus TM, Jaworsky DE, Frehywot GL et al (2000) Reduced food intake and body weight in mice treated with fatty acid synthase inhibitors. Science (New York) 288:2379-2381 
11. Kumar MV, Shimokawa T, Nagy TR, Lane MD (2002) Differential effects of a centrally acting fatty acid synthase inhibitor in lean and obese mice. Proc Natl Acad Sci U S A 99:1921-1925

12. Kim EK, Miller I, Landree LE et al (2002) Expression of FAS within hypothalamic neurons: a model for decreased food intake after C75 treatment. Am J Physiol Endocrinol Metab 283:E867-E879

13. Kim EK, Miller I, Aja S et al (2004) C75, a fatty acid synthase inhibitor, reduces food intake via hypothalamic AMP-activated protein kinase. J Biol Chem 279:19970-19976

14. Bonnefont JP, Djouadi F, Prip-Buus C, Gobin S, Munnich A, Bastin J (2004) Carnitine palmitoyltransferases 1 and 2: biochemical, molecular and medical aspects. Mol Aspects Med 25:495-520

15. Pocai A, Lam TK, Obici S et al (2006) Restoration of hypothalamic lipid sensing normalizes energy and glucose homeostasis in overfed rats. J Clin Invest 116:1081-1091

16. Obici S, Feng Z, Arduini A, Conti R, Rossetti L (2003) Inhibition of hypothalamic carnitine palmitoyltransferase-1 decreases food intake and glucose production. Nat Med 9:756-761

17. He W, Lam TK, Obici S, Rossetti L (2006) Molecular disruption of hypothalamic nutrient sensing induces obesity. Nat Neurosci 9:227-233

18. Price N, van der Leij F, Jackson V et al (2002) A novel brainexpressed protein related to carnitine palmitoyltransferase I. Genomics 80:433-442

19. Lavrentyev EN, Matta SG, Cook GA (2004) Expression of three carnitine palmitoyltransferase-I isoforms in 10 regions of the rat brain during feeding, fasting, and diabetes. Biochem Biophys Res Commun 315:174-178

20. Wolfgang MJ, Kurama T, Dai Y et al (2006) The brain-specific carnitine palmitoyltransferase-1c regulates energy homeostasis. Proc Natl Acad Sci U S A 103:7282-7287

21. Sierra AY, Gratacos E, Carrasco P et al (2008) CPT1c is localized in endoplasmic reticulum of neurons and has carnitine palmitoyltransferase activity. J Biol Chem 283:6878-6885

22. Ji S, You Y, Kerner J et al (2008) Homozygous carnitine palmitoyltransferase $1 \mathrm{~b}$ (muscle isoform) deficiency is lethal in the mouse. Molec Genet Metab 93:314-322

23. Nyman LR, Cox KB, Hoppel CL et al (2005) Homozygous carnitine palmitoyltransferase 1a (liver isoform) deficiency is lethal in the mouse. Molec Genet Metab 86:179-187

24. Wolfgang MJ, Cha SH, Millington DS et al (2008) Brain-specific carnitine palmitoyl-transferase-1c: role in CNS fatty acid metabolism, food intake, and body weight. J Neurochem 105:1550-1559

25. Dai Y, Wolfgang MJ, Cha SH, Lane MD (2007) Localization and effect of ectopic expression of CPT1c in CNS feeding centers. Biochem Biophys Res Commun 359:469-474

26. Lin J, Wu PH, Tarr PT et al (2004) Defects in adaptive energy metabolism with CNS-linked hyperactivity in PGC-1alpha null mice. Cell 119:121-135

27. Miyake K, Ogawa W, Matsumoto M, Nakamura T, Sakaue H, Kasuga M (2002) Hyperinsulinemia, glucose intolerance, and dyslipidemia induced by acute inhibition of phosphoinositide 3-kinase signaling in the liver. J Clin Invest 110:1483-1491

28. Barazzoni R, Bosutti A, Stebel M et al (2005) Ghrelin regulates mitochondrial-lipid metabolism gene expression and tissue fat distribution in liver and skeletal muscle. Am J Physiol Endocrinol Metab 288:E228-E235

29. Neschen S, Moore I, Regittnig W et al (2002) Contrasting effects of fish oil and safflower oil on hepatic peroxisomal and tissue lipid content. Am J Physiol Endocrinol Metab 282:E395-E401

30. Zisman A, Peroni OD, Abel ED et al (2000) Targeted disruption of the glucose transporter 4 selectively in muscle causes insulin resistance and glucose intolerance. Nat Med 6: 924-928

31. Liu L, Zhang Y, Chen N, Shi X, Tsang B, Yu YH (2007) Upregulation of myocellular DGAT1 augments triglyceride synthesis in skeletal muscle and protects against fat-induced insulin resistance. J Clin Invest 117:1679-1689

32. Cha SH, Hu Z, Chohnan S, Lane MD (2005) Inhibition of hypothalamic fatty acid synthase triggers rapid activation of fatty acid oxidation in skeletal muscle. Proc Natl Acad Sci U S A 102:14557-14562

33. McGarry JD, Mills SE, Long CS, Foster DW (1983) Observations on the affinity for carnitine, and malonyl-CoA sensitivity, of carnitine palmitoyltransferase $\mathrm{I}$ in animal and human tissues. Demonstration of the presence of malonyl-CoA in non-hepatic tissues of the rat. Biochem J 214:21-28

34. Jackson VN, Cameron JM, Zammit VA, Price NT (1999) Sequencing and functional expression of the malonyl-CoAsensitive carnitine palmitoyltransferase from Drosophila melanogaster. Biochem J 341:483-489

35. Kolodziej MP, Zammit VA (1990) Sensitivity of inhibition of rat liver mitochondrial outer-membrane carnitine palmitoyltransferase by malonyl-CoA to chemical- and temperature-induced changes in membrane fluidity. Biochem J 272:421-425

36. Cha SH, Rodgers JT, Puigserver P, Chohnan S, Lane MD (2006) Hypothalamic malonyl-CoA triggers mitochondrial biogenesis and oxidative gene expression in skeletal muscle: role of PGC1alpha. Proc Natl Acad Sci U S A 103:15410-15415

37. Dobbins RL, Szczepaniak LS, Bentley B, Esser V, Myhill J, McGarry JD (2001) Prolonged inhibition of muscle carnitine palmitoyltransferase-1 promotes intramyocellular lipid accumulation and insulin resistance in rats. Diabetes 50:123-130

38. Sebastian D, Herrero L, Serra D, Asins G, Hegardt FG (2007) CPT I overexpression protects L6E9 muscle cells from fatty acid-induced insulin resistance. Am J Physiol Endocrinol Metab 292:E677-E686

39. Kelley DE, Mintun MA, Watkins SC et al (1996) The effect of non-insulin-dependent diabetes mellitus and obesity on glucose transport and phosphorylation in skeletal muscle. J Clin Invest 97:2705-2713

40. Cline GW, Petersen KF, Krssak M et al (1999) Impaired glucose transport as a cause of decreased insulin-stimulated muscle glycogen synthesis in type 2 diabetes. N Engl J Med 341:240-246

41. Obici S, Feng Z, Morgan K, Stein D, Karkanias G, Rossetti L (2002) Central administration of oleic acid inhibits glucose production and food intake. Diabetes 51:271-275

42. Zhang D, Liu ZX, Choi CS et al (2007) Mitochondrial dysfunction due to long-chain Acyl-CoA dehydrogenase deficiency causes hepatic steatosis and hepatic insulin resistance. Proc Natl Acad Sci U S A 104:17075-17080 